\title{
The Aggregation of Striped Nanoparticles in Mixed Phospholipid Bilayers
}

\author{
Sang Young Noh, ${ }^{*, \dagger}$ 丹 Anthony Nash, ${ }^{*,+, \uparrow}$ and Rebecca Notman, ${ }^{*, \dagger}$ \\ $\dagger$ Department of Chemistry, University of Warwick \\ $\ddagger$ Nuffield Department of Clinical Neurosciences, University of Oxford \\ ฯ Contributed equally to this work
}

E-mail: s.y.noh@warwick.ac.uk; anthony.nash@dpag.ox.ac.uk; r.notman@warwick.ac.uk

\begin{abstract}
The unique and modifiable properties of nanoparticles present opportunities as targeted vector drug delivery mechanisms. Nanoparticles functionalized with key surface ligands have been shown to pass through phospholipid bilayers without becoming trapped or causing localised cellular disruption. However, the effect of ligandfunctionalized nanoparticles on the multi-component phospholipid bilayer is not clearly understood. This study investigates the structural properties of mixed bilayers in the presence of multiple ligand-functionalized nanoparticles. We show how: nanoparticles can aggregate near unsaturated regions and act as lineactants near phase-separated phospholipid boundary domains; the presence of ligand-functionalized nanoparticles drives bilayer phase-separation; and that the heterogeneous components of a phospholipid bilayer play a significant part in the lateral organization of nanoparticles.
\end{abstract}

\section{Keywords}

Nanoparticle, Bilayers, Molecular Dynamics, Drug Delivery, Phase Separation 


\section{Introduction}

Novel drug delivery mechanisms in biological environments have been the subject of intense study in recent years. ${ }^{1-6}$ For small drug molecules, the main barrier to efficient delivery is typically the cell membrane. ${ }^{7,8}$ The rate of passive diffusion depends on the concentration gradient between the aqueous exterior and the cell interior. ${ }^{8}$ In effect, this means that an excessive injection of drug molecules may be required and it may yet result in an inefficient delivery of drug molecules. ${ }^{8}$ In addition to the aforementioned obstacle to drug delivery, membrane proteins can act as effective barriers to a vast majority of molecules. For example, the membrane protein P-glycoprotein acts as a molecular pump expelling drug molecules from the inter-cellular space to the extent that it has been implicated in multidrug resistance of cancer cells. ${ }^{9}$

The use of nanoparticles (NPs) has been of increasing interest due to their potential for efficient, targeted drug delivery. NPs have demonstrated a level of effectiveness through multiple applications in targeted therapy. ${ }^{5,10}$ For example, Bozdaganyan et al., demonstrated that hydrophilic functionalized surfaces of fullerene can act as an antioxidant. ${ }^{11}$ However, there are a number if outstanding issues and a clear distinction between a therapeutic agent and an agent that induces nanotoxicity has often been difficult to make. ${ }^{6}$

In order to understand better the interactions of NPs with the lipid membranes, it is important to consider the multicomponent nature of the membrane, as the individual components of the bilayer are sensitively tuned to maintain the semi-permeable environment. For example, phospholipid molecules, a constituent part of the cellular membrane bilayer, impart their own properties onto the assembled bilayer through different degrees of saturation of the tailgroups, variations in tail length, and/or changes in headgroup functionality, affecting

both structure and structural phase, ${ }^{12-14}$ ranging from stable yet fluid-like disordered phases to highly ordered environments.

In addition, membrane proteins and other lipid molecules such as sterol and sphingolipidlike structures, are known to change the properties of the local phospholipid composition. ${ }^{15}$ 
For example, in sufficient concentration, cholesterol is known to reduce the curvature of the membrane. ${ }^{16}$ This indicates that the local composition of membranes has a significant affect on the mechanical properties of the phospholipid bilayer. With this in mind, an ideal targeted drug delivery mechanism must be designed such that it causes minimal disruption to the membrane, while also being able to freely traverse through the lipid bilayer.

Monolayer-protected NPs, collectively identified as ligand-functionalized NPs, are a candidate of particular interest for drug delivery due to the large variety of pharmacological and structural properties they can exhibit; for example, a NP with an inert gold core can be functionalized with thiolated ligands, which in turn can be functionalized with therapeutic drug molecules such as the chemotherapeutic agent Paclitaxel. ${ }^{17-20}$ Chemically optimized NPs allow the delivery of hydrophilic drug molecules through the hydrophobic region of the phospholipid membrane, which is the primary hindrance towards the effective delivery of drug molecules. ${ }^{21-23}$

The effects of the NP ligands on the local phospholipid arrangement has been the subject of a number of studies. For example, amphiphilic surface ligands result in spontaneous adsorption of the NP into the bilayer via an initial attraction between the bilayer phospholipid headgroups and the NP surface ligands, followed by hydrophobic interactions between the NP surface ligands and bilayer phospholipid tail groups. ${ }^{24,25}$ Studies on model membranes have shown how hydrophilic ligand-functionalized NPs are stabilized through a snorkelled configuration, where ligands adjust to maximise the hydrophobic and hydrophilic contacts with the phospholipid environment and the phospholipid-water interface. ${ }^{25}$ Katz and Van Lehn demonstrated that the snorkelling process is driven by minimising the exposure of the hydrophobic bilayer regions around the NPs. ${ }^{24,26}$ In particular, the higher the rigidity of the functionalized ligands on the NP surface the greater the free energy cost of such snorkelling and of NP insertion through hydrophobic contacts. Jackson et al found that by using a mixture of charged and hydrophobic NP surface-bound ligands it was possible to design a NP that could translocate the bilayer structure whilst minimising local disruption. ${ }^{27}$ Head- 
group charge can also modulate the insertion mechanism of the NP dramatically as seen from coarse-grained simulations of cationic, hydrophobic and anionic NPs through a mixed-charge bilayer (composed of symmetric and asymmetric negatively charged phospholipids). ${ }^{28}$ By increasing the surface charge density of the NP there is an increase in contact area between the phospholipid headgroups with the charged ligands on the NP surface, followed by the mediation of NP insertion through favourable hydrophobic contacts between the phospholipid tails and the NP ligands.

It remains to be seen whether the ligand-functionalized NPs alter the nature of the heterogeneous phospholipid bilayers. Rigid NPs of hydrophobic surface properties have demonstrated an effect on bilayer phase-separation. For example, Barnoud et al. demonstrated through mixtures of aliphatic and aromatic hydrophobic particulates and NPs in mixed DPPC, DUPC and cholesterol bilayers that aliphatic compounds aggregate near the domain interface, while aromatic compounds integrate to the disordered regions and stabilise the phases. $^{29}$

The phase-separated nature of a mixed phospholipid bilayer remains contentious, but growing evidence suggests that appropriate NP surface chemistry contributes to bilayer phase separation. Davis et al. showed that the phase separation into a mixed liquid-ordered/liquiddisordered phase is primarily due to the different interactions between the tail beads ${ }^{30}$ and sterol species. Another factor to consider is the cholesterol-induced structural order of the surrounding saturated phospholipids, which can induce significant order in the saturated lipid phase resulting in an increase in the rate of phase separation. Hence, this would suggest that conformational entropy of lipid/sterol species plays a significant factor in the phase separation process. ${ }^{31}$

In this work, we use molecular dynamics (MD) simulations to explore the effect of striped NPs on the properties of a bilayer composed of a mixture of saturated and unsaturated phospholipids and cholesterol. The striped topology for the NP was chosen as it has been demonstrated that such a topology provides the crticial amphiphilic property that ${ }^{19,32}$ can 
both traverse the hydrophilic solvent/bilayer exterior and the hydrophobic interior. Hence, the possibility of interaction of such NPs in the presence of domain-like structures within the bilayer is greatly increased. We have focused on two bilayer mixtures and one monophospholipid bilayer with a striped hydrophilic-hydrophobic NP present. We focused our attention on the effect of a singular NP and then multiple NPs on the phase separation of the bilayer.

\section{Methods}

\section{Simulation Details}

Coarse-grained MD simulations were performed using GROMACS-5.0.1. ${ }^{33,34}$ The short-range neighbour interaction list cut-off was fixed to $1.4 \mathrm{~nm}$ and updated every 10 steps. Nonbonded intermolecular interactions were described by a Lennard-Jones potential with the potential shifted to zero at $1.2 \mathrm{~nm}$. The pressure was set to 1 atm and controlled using the semi-isotropic Parinello-Rahman scheme. ${ }^{35-37}$ The temperature set to $323 \mathrm{~K}$ and regulated using the Berendsen coupling scheme. Both schemes had a relaxation time of 1.25 ps. This

temperature ensured the formation of phospholipid microdomain in the model bilayers. ${ }^{30,38,39}$ Equilibration simulations were performed for $100 \mathrm{~ns}$ and production run simulations for $5 \mu \mathrm{s}$. An integration time-step of 0.01 ps was used throughout. Frames were recorded every $1 \times 10^{6}$ steps for analysis. Additional simulation parameters and equilibrium information is provided in the supplementary information.

\section{Force-Field Details}

Atoms were encoded and unified into coarse-grained (CG) beads using the MARTINI forcefield. ${ }^{40}$ Each bead represents up to four atoms and are unified into one of four types: polar, nonpolar, apolar, or charged, corresponding to the labels P, N, C, and Q, respectively. Bead types used for each lipids are shown in Figure 1(a). 


\section{Model Construction}

The initial bilayer conformations were constructed using the INSANE lipodomics (INSert membraNE) tool. ${ }^{41}$ The INSANE algorithm provides a convenient way to adjust the size and composition of a MARTINI model bilayer and the insertion of biomolecules and NPs. Model bilayers were constructed using cholesterol (CHOL) and three phospholipid species: dipalmitoylphosphatidylcholine (DPPC), saturated; decadienoylphosphatidylcholine (DUPC), double unsaturated; and dioctadecatrienoylphosphatidylcholine (DFPC), triple-unsaturated (Figure 1(a)). The three bilayer compositions used throughout were DPPC-CHOL, DPPCDFPC-CHOL and DPPC-DUPC-CHOL with two unit cell sizes. The first had dimensions of $15 \mathrm{~nm}^{2}$ and then replicated in the $x$ and $y$ dimension to create a $30 \mathrm{~nm}^{2}$ bilayer. The $z$ dimension was $9 \mathrm{~nm}$ in both systems. Each mixed bilayer contained $30 \%$ cholesterol.

The construction of the NP topology used packmol, ${ }^{42}$ where the initial topology was constructed using the micelle topology, following the packing strategy of Packmol. ${ }^{43}$ The innermost beads were treated as the inert metal atoms, and the closest metal beads were treated with harmonic bond potentials of $5000 \mathrm{~kJ} \mathrm{~mol}^{-1}$ to ensure its rigidity. A harmonic bond potential of $1250 \mathrm{~kJ} \mathrm{~mol}^{-1}$ with a bond length of $0.47 \mathrm{~nm}$ and a cosine angle potential set to $180^{\circ}$ with the harmonic potential of $25 \mathrm{~kJ} \mathrm{~mol}^{-1}$ was applied to all other bonds in the ligand. The striped ligand-functionalized NP $(\mathrm{S}-\mathrm{NP})^{44}$ was constructed using charged and hydrophobic ligands (Figure $1(\mathrm{~b})$ ). The $15 \mathrm{~nm}^{2}$ bilayer contained a single S-NP and the $30 \mathrm{~nm}^{2}$ bilayer contained four S-NPs. The S-NPs were positioned within the centre-ofgeometry of each bilayer. The choice in ligands was similar to models by Simonelli et al, ${ }^{45}$ in which ligands based on octanethiol ligands (hydrophobic) and mercapto-decanesulfonate ligands (anionic) were used. Furthermore, in earlier studies the MARTINI force field had been used to design a general ligand-functionalized charged/hydrophobic S-NP using the

hydrophobic C beads, and negatively charged Qa beads. ${ }^{46,47}$ The length of each ligand type was approximately $1.7 \mathrm{~nm}$, while the radius of the S-NP core was approximately $0.7 \mathrm{~nm}$ giving an approximate diameter of $4.1 \mathrm{~nm}$ (although, this can fluctuate due to interactions 
with the water and phospholipid environment).

Each bilayer-S-NP construct underwent a series of equilibration steps. Firstly, steepest descent minimisation was performed to resolve steric clashes. This was followed with a 100 ns simulation using the NVT ensemble (constant number of particles $(N)$, volume $(V)$ and temperature $(T)$ ) over an increasing timestep from 0.0001 ps to 0.01 ps using the velocity-verlet integration algorithm at 10 ns intervals using velocity preserved simulation restarts. ${ }^{48-51}$ The simulation was then continued for $6 \mu$ s using an NPT ensemble (constant number of particles $(N)$, pressure $(P)$ and temperature $(T))$. The first $1 \mu$ s of production simulation data was discarded from the analysis. The complete catalogue of simulations presented in this study are summarised in Table 1.

Table 2: Catalogue of simulations carried out, showing the initial bilayer size, number of S-NPs, the quantity of phospholipids and cholesterol, and the phospholipid ratio.

\begin{tabular}{c|c|c|c|c|c|c|c}
\hline Simulation & Bilayer Size & NPs & DPPC & DUPC & DFPC & CHOL & Ratio \\
\hline DPPC-CHOL-NP & $15 \mathrm{~nm}^{2}$ & 1 & 476 & 0 & 0 & 204 & $7: 3$ \\
\hline DPPC-DUPC-CHOL-NP & $15 \mathrm{~nm}^{2}$ & 1 & 272 & 204 & 0 & 204 & $4: 3: 3$ \\
\hline DPPC-DFPC-CHOL-NP & $15 \mathrm{~nm}^{2}$ & 1 & 261 & 0 & 195 & 195 & $4: 3: 3$ \\
\hline DPPC-CHOL & $30 \mathrm{~nm}^{2}$ & 0 & 1088 & 0 & 816 & 816 & $4: 3: 3$ \\
\hline DPPC-DUPC-CHOL & $30 \mathrm{~nm}^{2}$ & 0 & 1088 & 0 & 816 & 816 & $4: 3: 3$ \\
\hline DPPC-DFPC-CHOL & $30 \mathrm{~nm}^{2}$ & 0 & 1088 & 816 & 0 & 816 & $4: 3: 3$ \\
\hline DPPC-CHOL-4NP & $30 \mathrm{~nm}^{2}$ & 4 & 1906 & 0 & 0 & 816 & $7: 3$ \\
\hline DPPC-DUPC-CHOL-4NP & $30 \mathrm{~nm}^{2}$ & 4 & 1088 & 816 & 0 & 816 & $4: 3: 3$ \\
\hline DPPC-DFPC-CHOL-4NP & $30 \mathrm{~nm}^{2}$ & 4 & 1044 & 0 & 780 & 780 & $4: 3: 3$ \\
\hline
\end{tabular}

\section{Analysis}

The GROMACS tools developed by Castillo et $a l^{52}$ were used to calculate the phospholipid tail order parameter and average density. For these analyses, the simulation unit cell was divided into $1 \mathrm{~nm}^{2}$ cells over a 2D grid over the $x \times y$ plane. The phospholipid tail order parameter and the phospholipid density were averaged within each cell and reported.

Cartesian components of the pressure tensors were calculated using the custom GROMACS ${ }^{53,54}$ distribution. The simulation unit cell was divided into $1 \mathrm{~nm}^{2}$ cells across the 
(a)

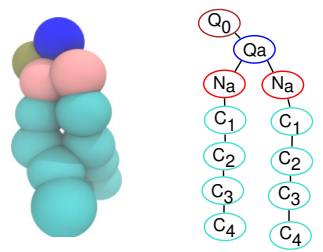

DPPC

(b)

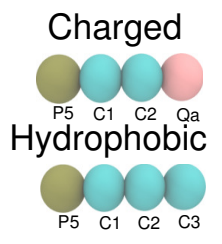

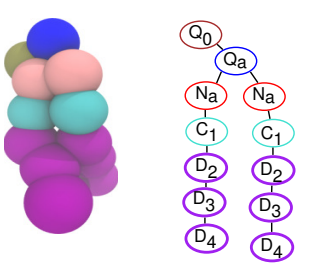

DFPC

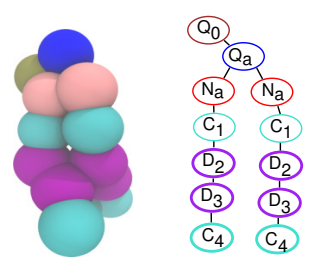

DUPC

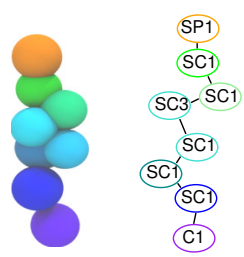

$\mathrm{CHOL}$

(c)

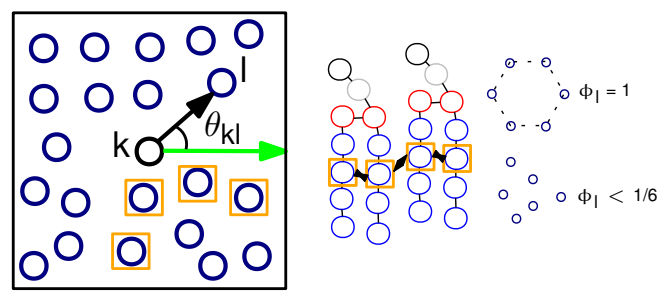

Figure 1: (a) The MARTINI bead composition of each phospholipid type and cholesterol. Cyan beads represent grouped saturated carbons, purple for 1-2 cis double bonds, pink denotes the glycerol linker region, the blue bead the phosphate, and brown represents choline. (b) The schematic of the S-NP, which consists of a hydrophobic center stripe capped with charged ligands. (c) The orderphobic effect is calculated between an arbitrary reference vector and the C3/D3 beads of neighbouring phospholipids.

bilayer normal and lateral plane (the lateral plane was chosen to be tangent to raft interfaces. Hence, the lateral plane would be either the $x$ or $y$ dimension and the normal would the $z$ dimension) and the local pressure tensors $P_{x x}, P_{y y}, P_{z z}$ were averaged across the the normal and lateral plane. From the pressure tensor components, we calculated the normal and lateral pressure across the profiles from the relations $P_{L}=-\left(\sigma_{x x}+\sigma_{y y}\right) / 2$ and $P_{N}=-\sigma_{z z}$. The line tension was calculated as $\gamma_{p}=\frac{1}{2}\left(P_{\mathrm{per}}-P_{\mathrm{par}}\right) L_{\mathrm{z}} L_{\mathrm{par}}$, where $P_{\mathrm{per}}$ and $P_{\text {per }}$ are pressure tensor components perpendicular and parallel to the phase interface, respectively. The $L_{\text {per }}$ and $L_{\text {par }}$ are the box dimensions in the bilayer normal direction and the bilayer lateral direction, respectively.

The impact of the orderphobic effect of the $\mathrm{NPs}^{55}$ was measured using the NelsonHalperin 2D bond-orientation order parameter. ${ }^{56,57}$ The average hexagonal packing parameters of the top and bottom phospholipid leaflets were calculated using 


$$
\phi_{l}=\left|\left(\frac{1}{6}\right) \sum_{l \in n n(k)}^{n} \exp \left(6 i \theta_{i j}\right)\right|^{2},
$$

where, $\theta_{i j}$ represents the angle between an arbitrary vector (in this case, we have used the $y$ unit vector) and the hexagonal vertices, as illustrated in Figure 1(c). The six closest phospholipid carbon-chain beads were used to calculate the extent of 2D-disorder around the S-NP. An equilibrium average of one represents a perfect hexagonal packing and zero represents disordered packing. The phospholipid beads $\mathrm{C} 2 A / \mathrm{C} 2 B$ and $\mathrm{D} 2 A / \mathrm{D} 2 B$ (where A and B labels represent the two hydrophobic tails) in the phospholipid tail groups were used as points of reference with respect to the arbitrary vector. ${ }^{58}$ All visualisations and analysis were implemented using a combination of VMD,${ }^{59}$ APLVoro ${ }^{60}$ and MDAnalysis, ${ }^{61}$ and in-house scripts.

\section{Results}

Each model was left to equilibrate for $1 \mu$ s (equilibration data provided in the Figure S1 of the supplementary information) followed by production runs for $5 \mu \mathrm{s}$. Representative time intervals of the trajectory were used to calculate average mass density and phospholipid tail order parameters, relative to the bilayer normal across the unit cell. In addition, 2D order parameters (phospholipid packing order) using the C2/D2 beads in the hydrophobic chain of DPPC, DUPC and DFPC phospholipids were calculated. Order parameters were used as a measurement of phospholipid order at the bilayer interface with the S-NP. Pressure tensor calculations were performed to account for any differences in line tension between bilayer constructs. Finally, the area-per-lipid (APL) and bilayer thickness was measured in the multi-S-NP simulations to identify phospholipid domain formations and bilayer deformations as the S-NPs approached a bilayer phase interface.

In the absence of S-NPs, both the DPPC-DUPC-CHOL simulations failed to reveal phospholipid phase separation, whereas phase separation in the DPPC-DFPC-CHOL model bi- 
layer was observed during the simulation (Figure S2).

\section{Single S-NP}

Single S-NPs in monomeric and two mixed-phospholipid bilayers, underwent production simulations for $5 \mu$ s as presented in Figure 2. Representative time frames for average mass density and phospholipid tail order calculations were taken at a point in which each system was seen to adopt a stable conformation (Figure 3 (a), (b) and (c)).

Phospholipid order (Figure 3 (a)) of the DPPC and cholesterol combination is lower among the annular phospholipids (those local to the S-NP) and assumes a phospholipid tail order indicative of a bulk DPPC bilayer, approximately $2 \mathrm{~nm}$ from the bilayer-S-NP interface. The average phospholipid density is greatest in the bulk bilayer and the presence of a S-NP alters the phospholipid density over a distance similar to the change in phospholipid tail order.

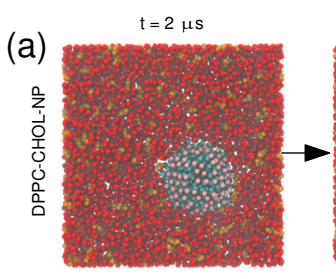

$t=3 \mu s$
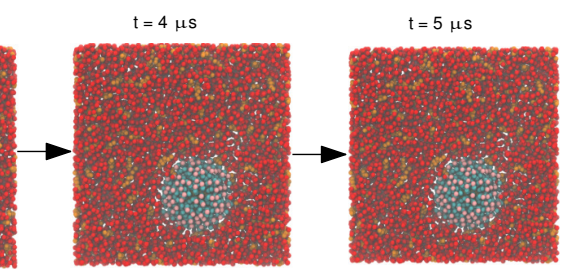

(b)

$t=2 \mu \mathrm{s}$

$\mathrm{t}=4 \mu \mathrm{s}$

$t=5 \mu \mathrm{s}$

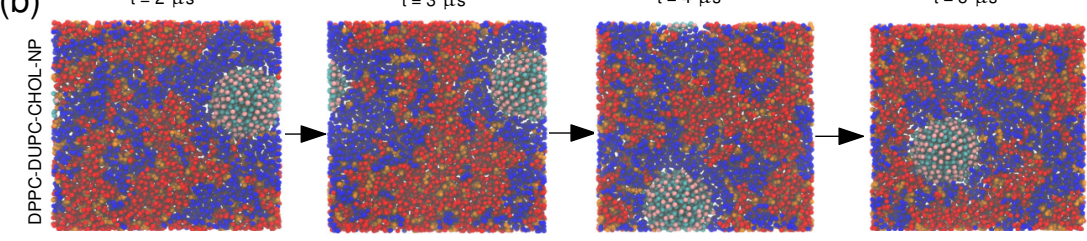

(c)

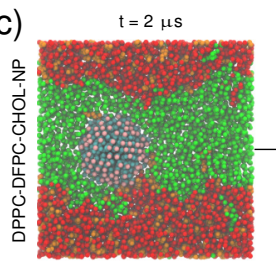

$t=3 \mu \mathrm{s}$

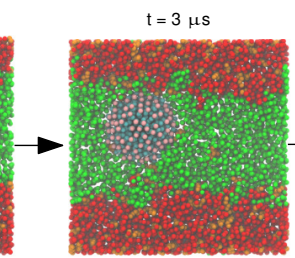

$\mathrm{t}=4 \mu \mathrm{s}$

$\mathrm{t}=5 \mu \mathrm{s}$

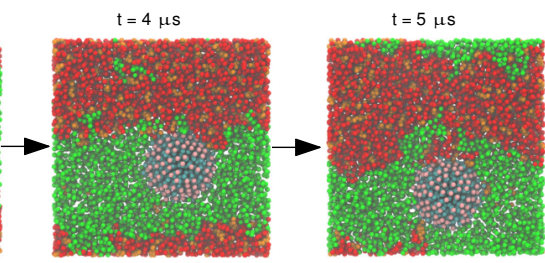

Figure 2: Time frames illustrating the evolution of each 1-NP bilayer system after equilibration. DPPC is red, DUPC blue, DFPC green and cholesterol is orange. Water has been removed for clarity.

The mixing of phospholipids DPPC with DUPC (Figure 2 (b)) resulted in saturated and 
unsaturated micro-domains formation within the first $100 \mathrm{~ns}$ with a consistent arrangement of DUPC phospholipids around the S-NP. The phospholipid tail order and mass density is similar to the values seen in the monophospholipid system, in that phospholipid order increases further from the S-NP, however, the difference between DUPC and DPPC introduces a greater degree of variance in phospholipid tail order. Within the simulation the phospholipid micro-domains consistently rearranged as phospholipids exchanged between domains. The mixing of phospholipids DPPC and DFPC (Figure 2 (c)), resulted in the formation of two phospholipid domains with cholesterol predominately found within the ordered DPPC domain. The DFPC domain, containing the S-NP, had significantly less ordered phospholipid tails that corresponds well with the distribution of phospholipid mass density. The DPPC-DFPC interface presents a gradient in phospholipid tail order.

The 2D bond order (Figure $3(\mathrm{~d})$ ) is a measurement of phospholipid packing order and was calculated from the phospholipid-S-NP interface into the bulk bilayer. The packing order for the monophospholipid system, DPPC-CHOL, converges to a bulk arrangement within the first $0.5 \mathrm{~nm}$ from the phospholipid-NP interface. DPPC-DUPC-CHOL presents a sporadic arrangement of phospholipid packing, indicative of the formation of many small phospholipidmicrodomains with DUPC lipids present local to the S-NP. The phospholipid order stabilises after $4 \mathrm{~nm}$ from the phospholipid-S-NP interface. The phospholipid arrangement of DPPCDFPC-CHOL presents two intervals in phospholipid packing order, as a result from the formation of the two phospholipid micro-domains.

The time-average phospholipid order parameter was measured from the centre-of-mass (COM) of the S-NP (Figure $3(\mathrm{e})$ ). The low parameter values over the initial $2 \mathrm{~nm}$ are due to the measurement being made from the S-NP COM. The large degree of variance at $2 \mathrm{~nm}$ is due to undulations in the S-NP-bound ligands, promoting a degree of exchange in phospholipids within this narrow space. The phospholipid order parameter of the unsaturated phospholipids, DUPC and DFPC, is consistently lower than the saturated DPPC over the breadth of the unit cell. Whilst the order parameter of the saturated phospholipids remained 
constant, the order parameter for DPPC steadily increased the further from the S-NP.

We calculated the $x, y$, and $z$ components of the lateral and normal bilayer pressure profiles averaged through the bilayer interface ( $x$ as defined by the distinct bilayer micro-domain interface formation in DPPC-DFPC-CHOL) across each simulation and found distinct pressure profiles dependent on the bilayer composition. In the lateral bilayer pressure (Figure 4 (a)), the $\mathrm{P}_{y y}$ and $\mathrm{P}_{z z}$ components in each bilayer fluctuated around 0 bar. Yet, the $\mathrm{P}_{x x}$ component (dimension parallel to the bilayer), presented a profile dependent on the S-NP and the phospholipid bilayer composition. In DPPC-CHOL bilayer, pressure peaks are local to the phospholipid-S-NP interface before decreasing by approximately 350 bar (7-12 nm), whilst in bulk the pressure fluctuates within the range -325 to -500 bar. The pressure peaks on either side of the S-NP in DPPC-DUPC-CHOL are not present, rather, the pressure peaks local to the S-NP adopt pressure similar to the bulk-bilayer (-325 to -430 bar). Yet, a +100 bar increase in pressure can be discerned from the clear peak local to the S-NP and after a drop in pressure at the phospholipid-S-NP interface.

With the formation of two distinct membrane micro-domains in the DPPC-DFPC-CHOL bilayer, the DPPC and cholesterol domain demonstrates a flat $\mathrm{P}_{x x}$ pressure profile which gradually increases (from 10-12.5 nm) in pressure by approximately +150 bar as DPPC, DFPC, cholesterol and the S-NP interact. The lowest pressure in the lateral range is indicative to the presence of a higher concentration in DPPC phospholipids. At the clear DPPC to DFPC interface, the pressures decrease by an insignificant amount before climbing to -300 bar in the bulk of the DFPC domain $(2.5 \mathrm{~nm})$. Finally, the pressure drops in the annular DFPC phospholipids.

The pressure profiles calculated normal to the bilayer (Figure 4 (b)), fluctuate consistently around 0 bar in the $\mathrm{P}_{y y}$ and $\mathrm{P}_{z z}$ component. Averaged along the $x$ axis, there are two distinct peaks of negative pressure approximately level with the hydrophilic phospholipid head groups. The pressure increases in the bilayer core. The lower pressure peaks in the monophospholipid DPPC and cholesterol bilayer construct are due to the homogeneous 
phospholipid composition. The lateral $\left(\mathrm{P}_{y y}\right.$ and $\left.\mathrm{P}_{x x}\right)$ and normal pressure profiles averaged along the bilayer normal (Figure 4 (c)), remain consistent with the trend of their individual pressure tensors. From the pressure tensor profiles, we can estimate the line tension between the phases, where the primary difference comes from $\mathrm{P}_{x x}(100$ bar) for the DPPCDUPC-CHOL system (approximately $6.83 \times 10^{-10} \mathrm{~N}$ ), and approximately 200 bar for the DPPC-DFPC-CHOL system (approximately $1.36 \times 10^{-9} \mathrm{~N}$ ). 
(a) DPPC-CHOL-NP
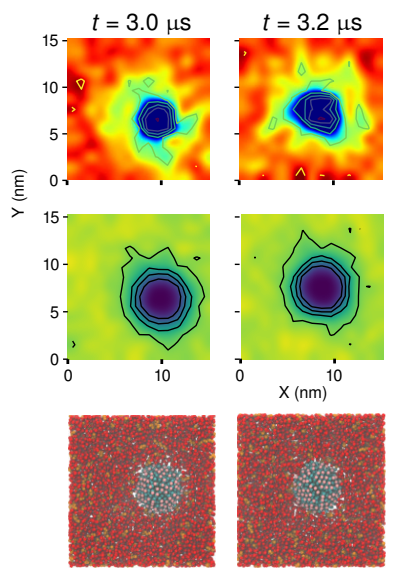

(d)
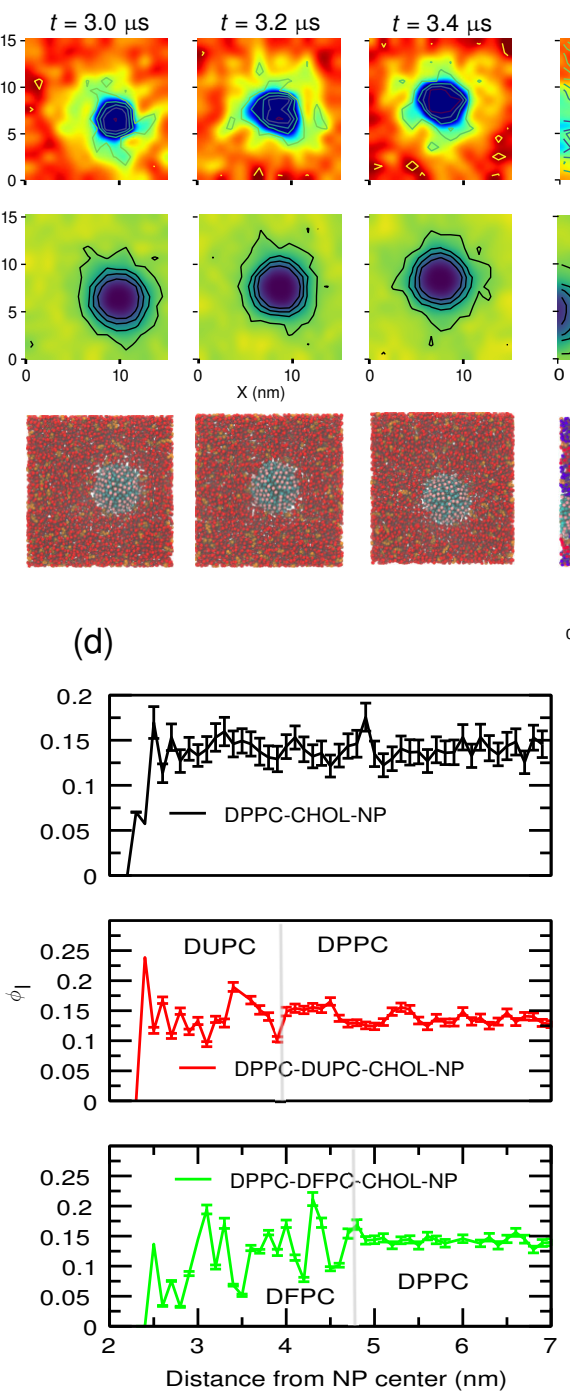

(b) DPPC-DUPC-CHOL-NP
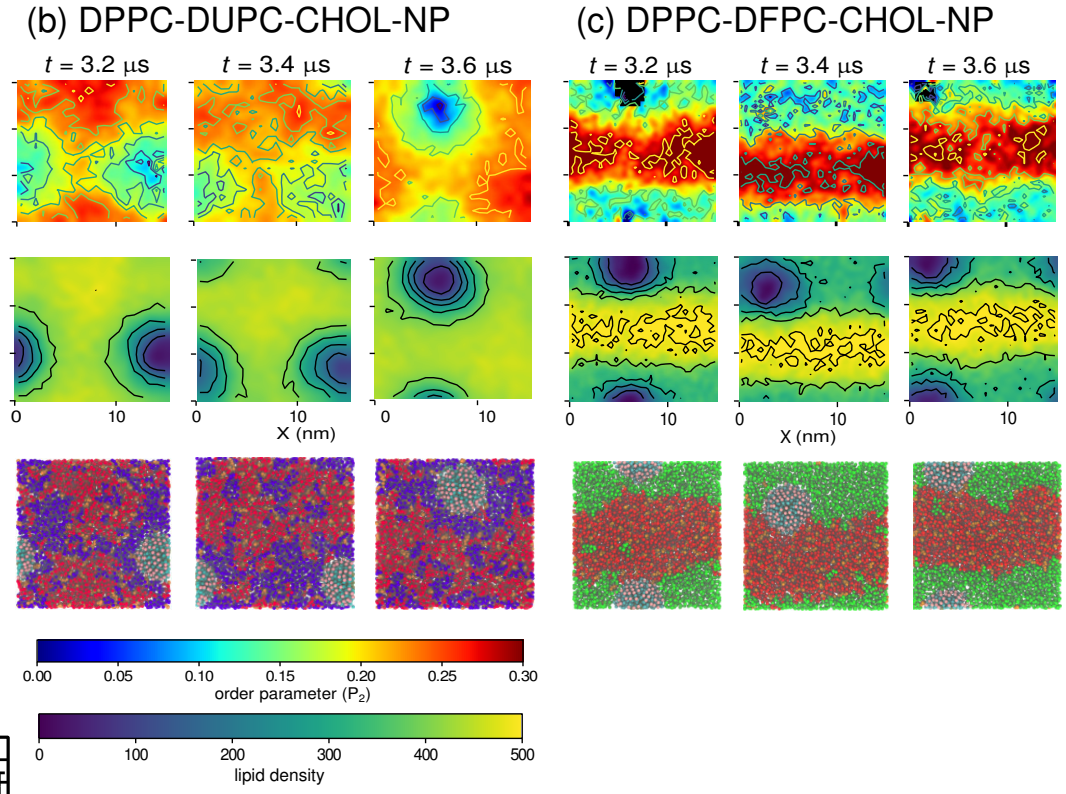

(e)

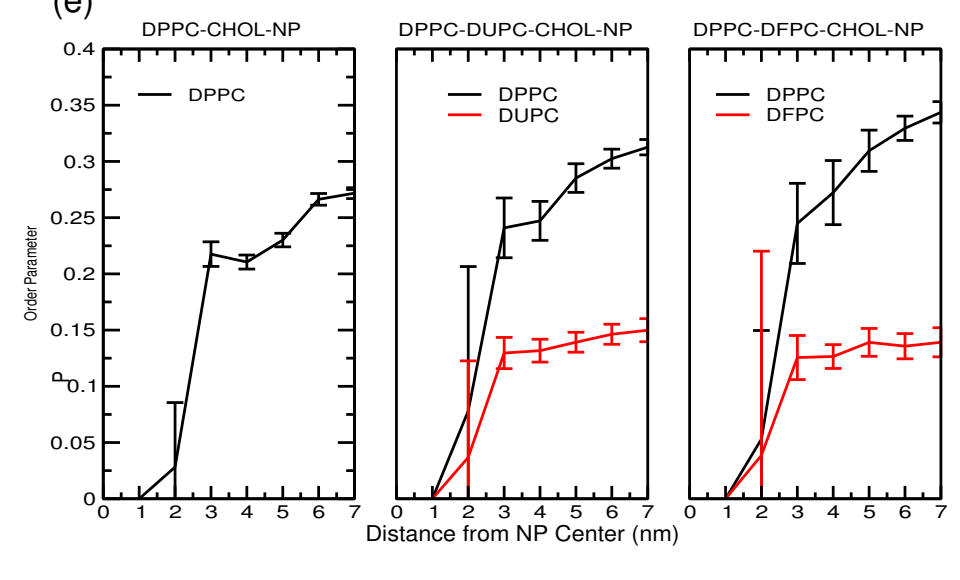

Figure 3: Three frames showing the phospholipid tail bond order (top) analysis and average mass density (middle) of the three 1-NP systems, (a) DPPC-CHOL, (b) DPPC-DUPCCHOL and (c) DPPC-DFPC-CHOL. Each frame was selected as a representative of the system at equilibrium. (d) The $2 \mathrm{D}$ bond order of the bilayer as a function of distance from the centre-of-mass of the NP. (e) The phospholipid tail bond order of individual phospholipid types as a function of distance from the centre-of-mass of the NP. Each data point was calculated as an average over 1000 ns. Error bars represent the standard error of the mean. 
(a)
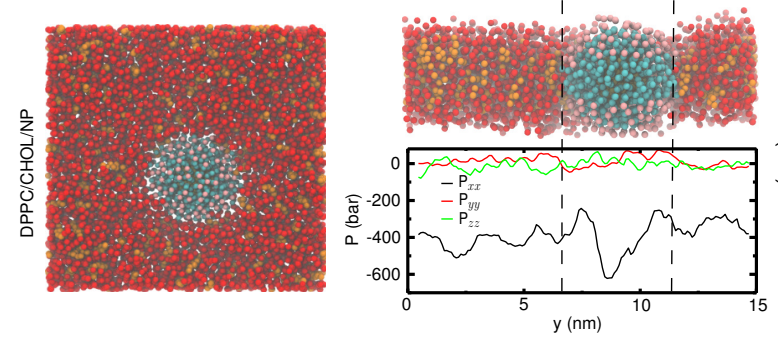

(b)
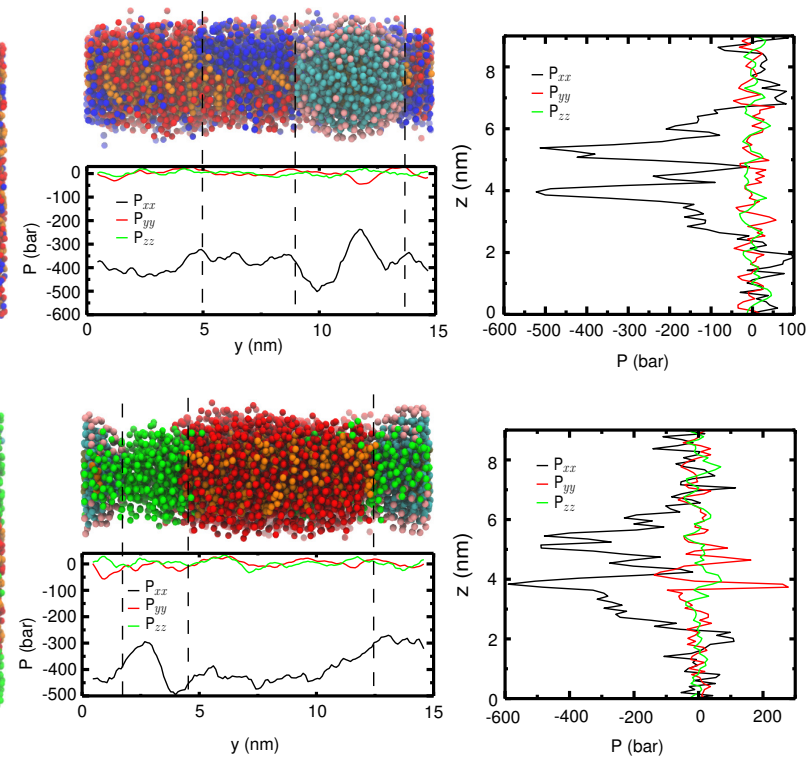

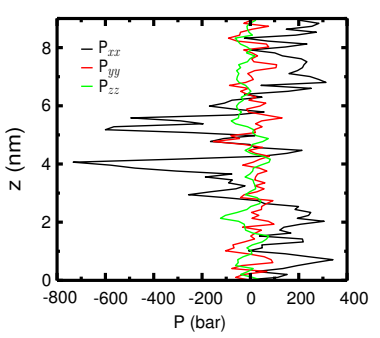

(c)
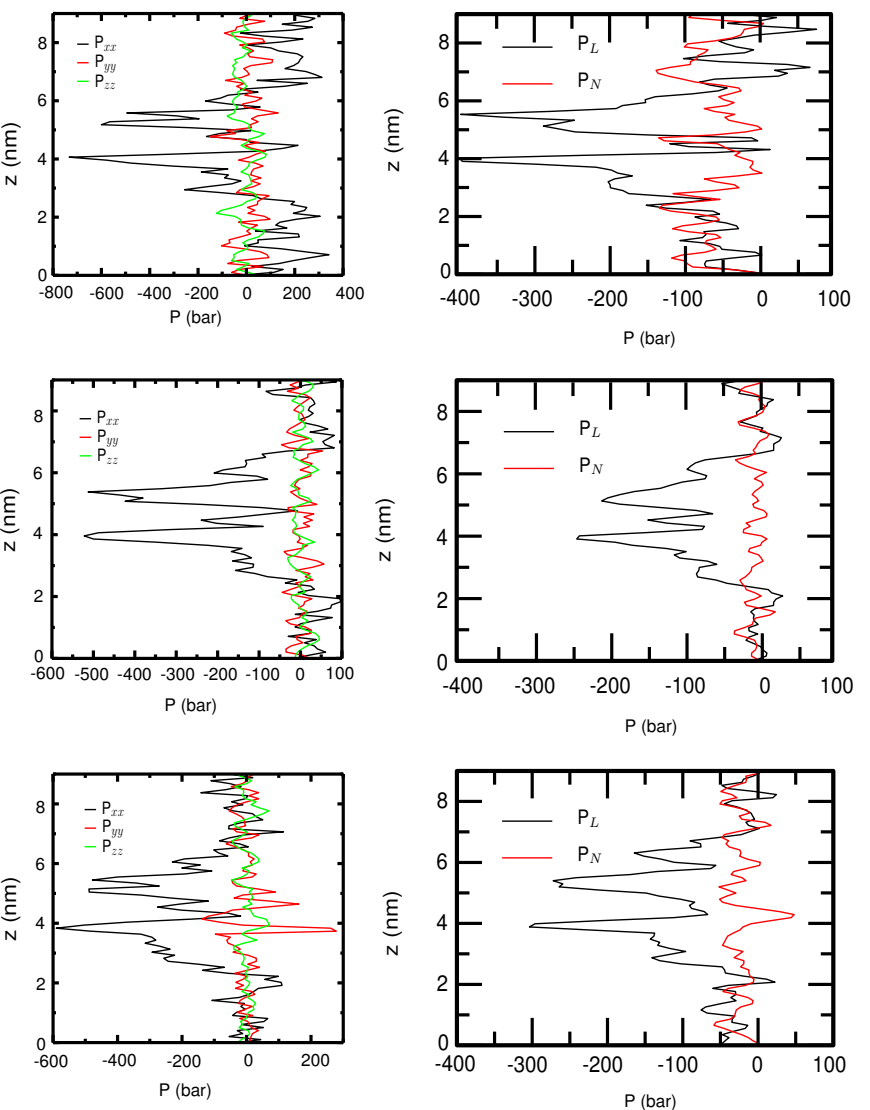

Figure 4: (a) and (b) show the pressure tensor extractions $\left(P_{x x}, P_{y y}, P_{z z}\right)$ along the bilayer lateral plane and the normal plane, for DPPC-CHOL, DPPC-DUPC-CHOL, and DPPCDFPC-CHOL, respectively and (c), the $P_{L}$ and $P_{N}$ for each bilayer along the bilayer tangent. 


\section{Multiple S-NPs}

The S-NP simulations, monomeric and mixed-phospholipid, underwent production runs for $5 \mu$ s. The average mass density and phospholipid order calculations were taken when each system was seen to adopt a stable conformation (Figure 5 (a), (b) and (c)).

In the phospholipid DPPC-CHOL simulation (Figure 6 (a)) a decreasing order parameter was observed local to the S-NPs, approximately $0.17 \mathrm{~nm}$ from the phospholipid-S-NP interface. As the S-NPs aggregate during the initial $100 \mathrm{~ns}$, the analysis of the phospholipid bilayer was performed over three frames which represent stable bilayer configurations. The average mass density was consistent throughout the bulk bilayer at approximately 400$500 \mathrm{~kg} \mathrm{~m}^{3}$, but dropped 250-300 $\mathrm{kg} \mathrm{m}^{3}$ within the local phospholipid arrangement. This difference demonstrates ordered packing in the bulk bilayer and disorder local to the S-NPs.

The DPPC-DUPC-CHOL simulations reveal a reduction in the phospholipid tail order parameter through a gathering of unsaturated DUPC phospholipids, whilst cholesterol migrated into the saturated DPPC regions. The average mass density remained consistent throughout the simulation, with a sharp decrease in density similar to the decrease in phospholipid tail order local to the S-NP aggregate.

During the simulation of the DPPC-DFPC-CHOL-4NP system, a DFPC phospholipid micro-domain formed within the initial $100 \mathrm{~ns}$ around the NPs, stabilising the S-NP aggregation whilst the complete phase separation as presented in Figure 6 (c) occurred. We see a gradient of order parameters between the unsaturated DFPC and the saturated DPPC domains. There is a clear mass order difference between the two phospholipid domains, with DPPC-CHOL showing the greater mass density compared to the DFPC microdomain. The difference in mass density at the phospholipid-NP interface is very slight, similar to the difference in phospholipid order, suggesting the favourable inclusion of the S-NPs in the DFPC microdomain.

The averaged APL and thickness for each species in each S-NP system are shown in figure 6 (d). For the DPPC-CHOL-4NP sample, for the area of cholesterol, we observed a 
value of $\sim 0.42 \mathrm{~nm}^{2}$, with the DPPC we observe a higher area of $\sim 0.52 \mathrm{~nm}^{2}$. The cholesterol thickness was $\sim 3.4 \mathrm{~nm}$, while the DPPC thickness was $\sim 4.2 \mathrm{~nm}$. The APL across the tri-component membranes molecules of DPPC-DUPC-CHOL-4NP remained consistent. Cholesterol had the smallest area $\left(\sim 0.45 \mathrm{~nm}^{2}\right)$, followed then by the saturated DPPC phospholipid $\left(\sim 0.525 \mathrm{~nm}^{2}\right)$ and finally, the DUPC phospholipid $\left(\sim 0.65 \mathrm{~nm}^{2}\right)$, which follows the kinked nature of the DUPC phospholipid allowing for a larger APL. The bilayer thickness as an average measurement between identical phospholipid types is quite clear; the saturated DPPC yields the greatest thickness $(\sim 4.2 \mathrm{~nm})$, followed by the unsaturated DUPC phospholipid $(\sim 3.95 \mathrm{~nm})$ and finally cholesterol $(\sim 3.4 \mathrm{~nm})$. The APL and bilayer thickness in the DPPC-DFPC-CHOL-4NP bilayer was also computed. The area of cholesterol and DPPC are very similar given their close packing $\left(\sim 0.42 \mathrm{~nm}^{2}\right.$ and $\sim 0.48 \mathrm{~nm}^{2}$ respectively $)$ with each other, whilst the two unsaturated hydrocarbon tails of DFPC show an increase in APL $\left(0.65 \mathrm{~nm}^{2}\right)$. With regard to thickness, we see that the cholesterol molecules and the DFPC phosphoplipids show similar ranges in lipid thickness $(\sim 3.6-\sim 3.8 \mathrm{~nm})$ and a larger thickness for the DPPC $(\sim 4.3 \mathrm{~nm})$. We also observe the effect of the S-NPs on the local

cholesterol density (Figure $6(\mathrm{e})$ ); we see a minimal change in the cholesterol density for the DPPC-CHOL-4NP system (with a peak value of approximately 1.1), whilst we see distinctive and decreasing densities of the cholesterol around the NPs in the DPPC-DUPC-CHOL-4NP and DPPC-DFPC-CHOL-4NP (where the values of the radial distribution function (RDF) decreases from approximately 0.8 to 0.4 (DPPC-DUPC-CHOL-4NP) and 0.5 to 0.2 (DPPCDFPC-CHOL-4NP)).

\section{Discussion}

In a previous experimental study, nanoparticles with mixed functionalization enter the bilayer interior through the interactions between the charged ligands and the hydrophilic headgroups of the bilayer. ${ }^{24}$ The subsequent 'snorkelling' of the local phospholipids induces a hydrophobic 

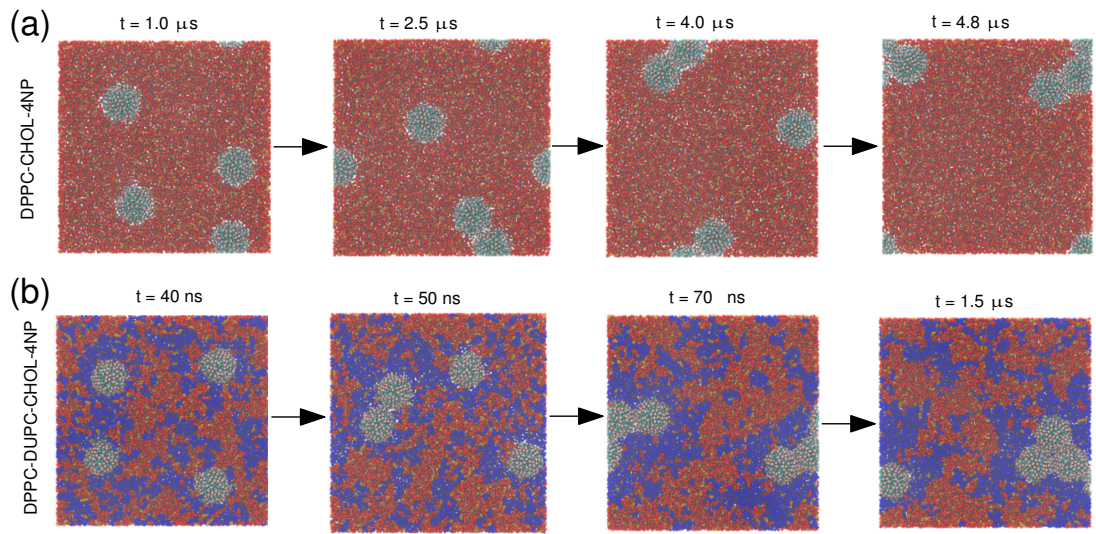

$\mathrm{t}=50 \mathrm{~ns}$

$\mathrm{t}=70 \mathrm{~ns}$

$\mathrm{t}=1.5 \mu \mathrm{s}$

(c)

$\mathrm{t}=10 \mathrm{~ns}$
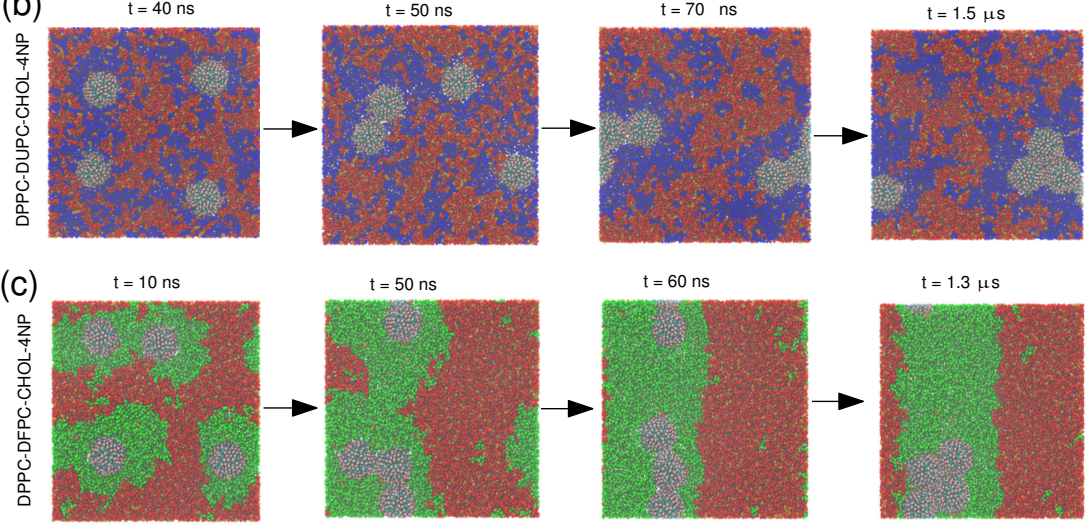

$\mathrm{t}=50 \mathrm{~ns}$

$\mathrm{t}=60 \mathrm{~ns}$

$\mathrm{t}=1.3 \mu \mathrm{s}$
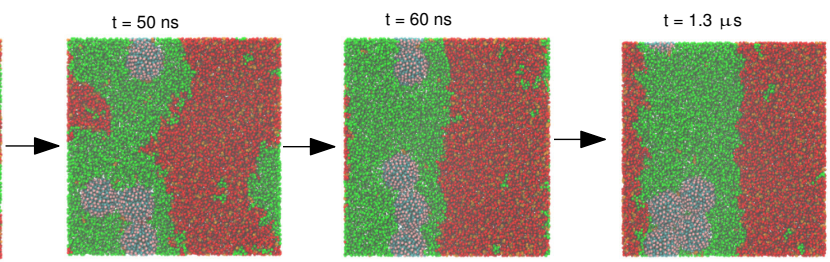

Figure 5: Single time frames illustrating the change to the bilayer and aggregation of the NPs as each system evolves. DPPC is red, DUPC blue, DFPC green and cholesterol is orange. Water has been removed for clarity.

mismatch between the snorkelled phospholipids and the rigid cholesterol molecules, resulting in the depletion of local cholesterol density near the NP region. ${ }^{46,47}$ From our single S-NP DPPC-DUPC-CHOL and DPPC-DFPC-CHOL simulations, we see the local depletion of cholesterol corresponding with phospholipids exchange between DPPC and either DUPC or DFPC, which in turn drives the formation of a bulk DPPC-CHOL region away from the S-NP. Risseleda et al showed that cholesterol in phase-separated bilayers is enthalpically driven to maximize levels of intermolecular contact with saturated phospholipids tails, ${ }^{38}$ which corresponds to an additional driver for the aggregation of unsaturated phospholipids near the S-NP. Our simulations indicate multiple distinct mechanisms for the aggregation of S-NPs, depending on the phospholipid composition. In the case of the DPPC-DUPC-CHOL and single S-NP, the cholesterol depletion drives the DUPC aggregation near the NPs, which in turn drives the increased fluidity of the S-NPs on an unsaturated domain. The equilibrium simulation snapshots reveal the formation of an unsaturated semi-circular domain near the SNP clusters (in the cases of DPPC-DUPC-CHOL and DPPC-DFPC-CHOL). As indicated by 
(a) DPPC-CHOL-4NP

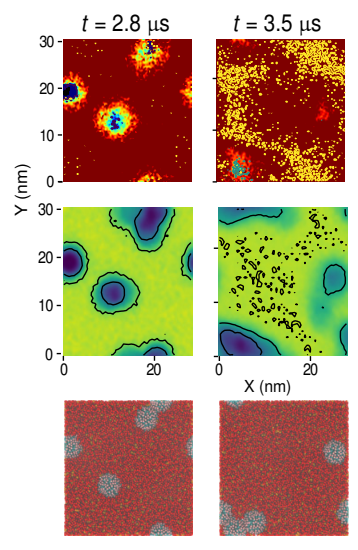

$t=4.8 \mu \mathrm{s}$
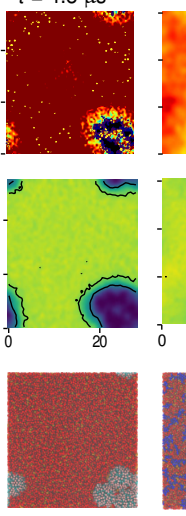

(b) DPPC-
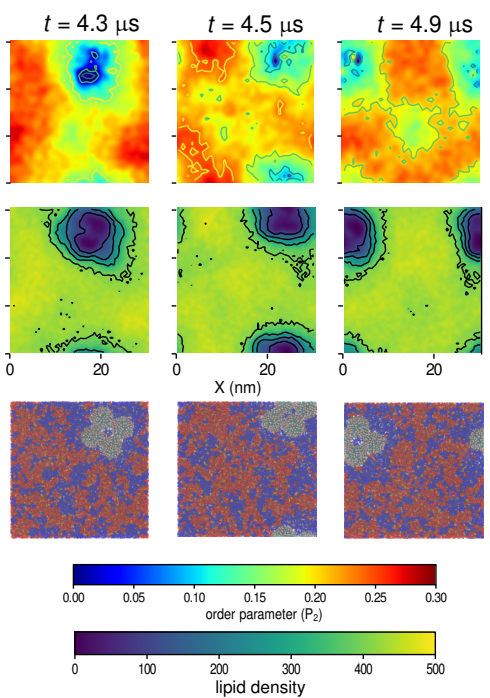

(d)

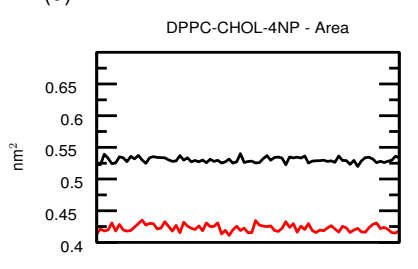

DPPC-DUPC-CHOL-4NP - Area
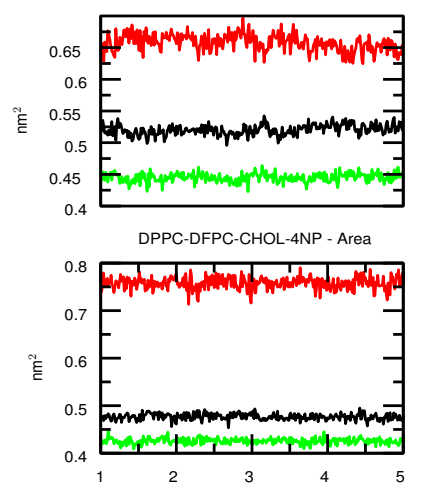

DPPC-CHOL-4NP - Thickness

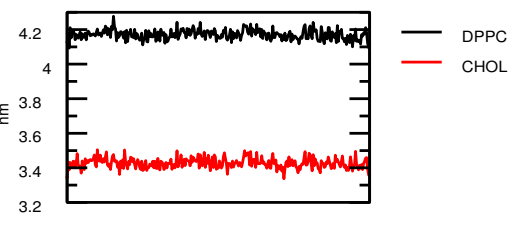

DPPC-DUPC-CHOL-4NP - Thickness
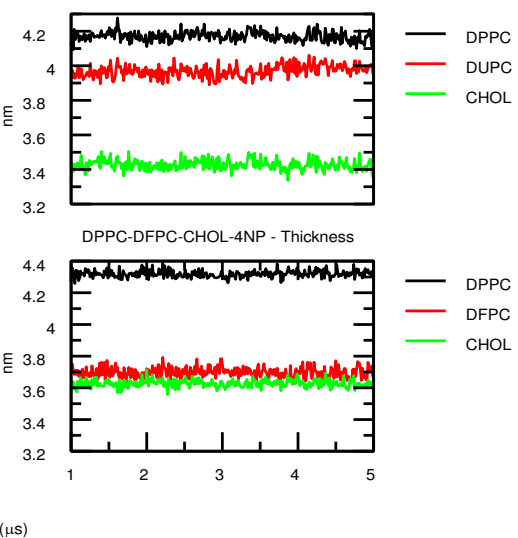

(c) DPPC-DFPC-CHOL-4NP

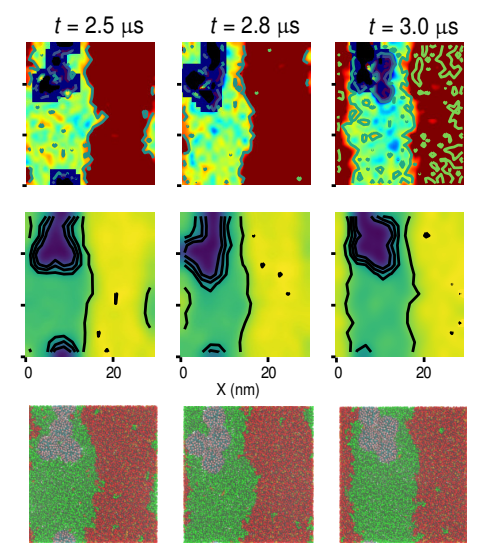

(e)

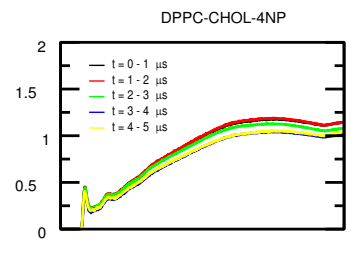

DPPC-DUPC-CHOL-4NP

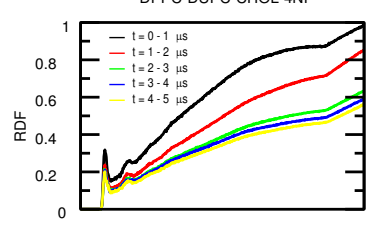

DPPC-DFPC-CHOL-4NP

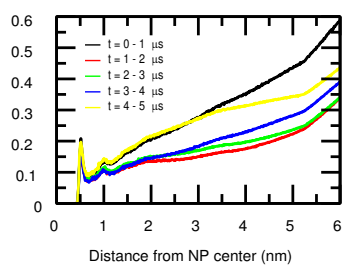

Figure 6: Analyses for the 4-NP systems for the DPPC-CHOL, DPPC-DUPC-CHOL and DPPC-DFPC-CHOL. (a), (b) and (c) show the order parameter and mass density heat maps for a series of configurations of the bilayer systems. (d) shows the area and thickness of the phospholipid bilayer for the DPPC-CHOL-4NP, DPPC-DUPC-CHOL-4NP and DPPCDFPC-CHOL-4NP systems. (e) shows the RDF profiles of the cholesterol molecules around the NPs, averaged in one $\mu$ s intervals over the $5 \mu$ s simulation time.

studies of mismatch energetics by $\mathrm{Hu}$ et al, ${ }^{62}$ domain formation before full phospholipid phase separation occurs when the hydrophobic mismatch is significant. The domain formation with the DPPC-DUPC-CHOL bilayer only occurs with the inclusion of the S-NP, while it is fully 
observable in both the NP/NP-absent case with the DPPC-DFPC-CHOL bilayer, indicating a greater hydrophobic mismatch between the DPPC-DFPC pair as compared to the DPPCDUPC pair.

For the cases where we have unsaturated mixed components (DUPC or DFPC), the partitioning of the NPs into the $l_{d}$ region is the key feature seen within our simulations, and it follows similar results as seen by previous studies of transmembrane helices. ${ }^{63}$ This has been attributed to the favourable enthalpic interactions between tightly packed $l_{o}$ phospholipids, which in turn drives the lateral sorting of protein helices. The packing in the $l_{o}$ phase (DPPCDPPC packing and DPPC-CHOL) is enthalpically favourable, and whilst the inclusion of the NPs into the $l_{d}$ phase would increase the global entropy of the mixed bilayer, the enthalpic penalty of disrupting the $l_{o}$ region prevents any aggregation of the NPs in the $l_{o}$ region.

In the case of the multiple S-NPs in the DPPC-CHOL bilayer, the absence of unsaturated components delays the formation of the energetically stable S-NP clover-like aggregation, compared to the DPPC-DUPC-CHOL/DPPC-DFPC-CHOL simulations. The effect of the shape of the NPs in the properties of aggregation can be seen to be analogous to those of membrane proteins; Parton et $a l^{64}$ demonstrated the effect of hydrophobic mismatch on $\alpha$-helical transmembrane (TM) proteins, and indicated that phospholipid demixing around the proximity of the TM proteins occur, resulting in the aggregation of phospholipids around the proteins where the contact between the hydrophobic chains and the hydrophobic tailgroups of the phospholipids was maximised. In the case of the DPPC-CHOL simulation, the phospholipid demixing around the NPs would be to displace the cholesterol molecules around the NP, which is enthalpically unfavourable.

Another interesting point of note is the difference between the structures of the aggregates observed here and of that in the simulation works by Angelikopoulos et al, ${ }^{46,47}$ which predicted a linear aggregate structure. The key difference was the increased length of the hydrophobic ligands (compared to the study by Angelikopoulos), which would suggest that the increased length of these ligands effectively increase the hydrophobic surface of the S-NP, 
which has two effects, namely to hinder the movement of the hydrophilic ligands to conform into a pseudo-snorkelling formation and increase the hydrophobic contacts between the phospholipids around the S-NPs. It was predicted that the main driver for the linear aggregation to change into a clover-like cluster was the orderphobic effect, ${ }^{55}$ which was observed to varying extents in our study. This phenomena closely follows the aggregation mechanism of transmembrane proteins and protein-mimics due to the orderphobic effect, which predicts the aggregation of protein-like structures due to the formation of a local order-disorder interface.

This analysis follows many other studies into membrane protein structures; Madsen et al demonstrated that the influenza A matrix 2 (M2) transmembrane protein induces local membrane remodelling by the aggregation of proteins. ${ }^{65,66}$ The M2 protein was shown to induce the local lowering of the order parameter of the end termini of the hydrophobic tails, for both 'stiff' and 'soft' phospholipids, analogous to DPPC ('stiff') phospholipid and DUPC/DFPC phospholipid ('soft') respectively, which is similar to the lowered phospholipid tail order parameters for the DPPC, DUPC and DFPC phospholipids in our simulations.

Ollila et al studied the pressure profile surrounding the mechanosensitive protein channel of Mycobacterium tuberculosis. The monolayers surrounding the protein ${ }^{67,68}$ show a lower lateral tension and the formation of spontaneous curvature due to this would cause the redistribution of lipids around the protein. Compared to our simulations, we see a variety of similar effect on the pressure tensor components near the lateral pressure of the NP. In the monophospholipid DPPC-CHOL bilayer, we see a distinct drop within the S-NP region, while in the mixed DPPC-DUPC-CHOL and DPPC-DFPC-CHOL bilayers, we observe a distinct 'buckle' in the lateral position of the S-NP or at the S-NP interface. This suggests that there are two effects of an S-NP at the phospholipid domain interface; firstly, the lowering of lateral pressure by the interaction of the S-NP with the phospholipid-domain interface, and secondly, the increase of lateral pressure due to a reorganization of phospholipids in monolayer regions further from the phospholipid domain region. In the DPPC-DUPC-CHOL case, we see the 
formation of small DUPC phospholipid microdomains in the vicinity of the S-NP region of approximately $2.0-2.5 \mathrm{~nm}$ radius. This corresponds to the highly fluctuating orderphobic region, seen in the 2.0-4.0 nm distance from the NP center. This analogous region is absent in the DPPC-DFPC-CHOL case, but we suggest that this is due to the linear domain boundary formed near the S-NP region, which would result in the collective average of the stable and fluctuating phases in the DPPC-CHOL and DFPC regions formed.

Following this, in the case of the DPPC-DUPC-CHOL simulation, the reduction of the line tension drives the aggregation of S-NP into a clover formation, while in the DPPCDFPC-CHOL case, we see that the rapid domain formation drives the clustered aggregation of S-NPs. Similar aggregation behaviour has been seen in transmembrane proteins. For example, Yoo et al ${ }^{69}$ demonstrated that for the association of gA proteins, two factors were noticeable, the compression of the phospholipids in between the proteins leading to a higher energy barrier of association, and the depletion of local phospholipids increasing the fluidity of the proteins. It was also observed that typically, clusters of at most four proteins were seen. When comparing to our study, we suggest that the local disordering of phospholipids around the S-NPs, induced by the hydrophobic mismatch between the DPPC and DUPC-DFPC and the favourable interaction between the DPPC and cholesterol, drives the aggregation of unsaturated phospholipids (DUPC-DFPC) within the vicinity of the S-NP. The subsequent aggregation between S-NPs is driven by the reduction of the energetically unfavourable domain interface between the $l_{d}$ and the $l_{o}$ in the DPPC-CHOL region.

\section{Conclusion}

We have simulated the integration of a hydrophilic-hydrophobic ligand functionalized S-NP in DPPC-DUPC-CHOL and DPPC-DFPC-CHOL mixed bilayers, and the monophospholipid bilayer DPPC-CHOL. The mixed bilayers were chosen to simulate near and certain phase separating systems. From this study, we have observed how an orderphobic effect 
is a driving force for S-NPs to merge with disordered regions in mixed bilayers. We have demonstrated that the hydrophobic mismatch between local phospholipids and the S-NPs drive aggregations of phospholipid species local to the S-NP region. We see that the effect is present in the case of the S-NPs, which in turn lowers local cholesterol density, resulting in the aggregation of unsaturated domains near the S-NP. This indicates that raft-like structures could significantly affect drug-delivery mechanisms of NPs.

While our study does not conclusively show that the hydrophobic tail group regions around the S-NPs are orderphobic according to the 2D-order parameter, it is clear that the order-disorder interface around the S-NP structures does exist. ${ }^{55,70}$ In addition, we see competition between S-NPs for the space to interact with the domain interfaces which encompass disorder/order boundaries. This allows the line tension to become an effective coordinating force for the aggregation of NPs. In summary, our study shows that the effect of ligand-functionalized S-NPs in mixed bilayers is dependent on the membrane composition, that orderphobic effect drives the aggregation of S-NPs, where the reduction of line tension effectively drives the S-NP aggregation.

\section{References}

1. Allen, T. M.; Cullis, P. R. Drug Delivery Systems: Entering the Mainstream. Science 2004, 303, 1818.

2. Blanco, E.; Shen, H.; Ferrari, M. Principles of nanoparticle design for overcoming biologial barriers to drug delivery. Nature Biotechnology 2015, 33, 941.

3. Jang, H. The Diverse Range of Possible Cell Membrane Interactions with Substrates: Drug Delivery, Interfaces, and Mobility. Molecules 2017, 22, 2197.

4. Kang, M.; Loverde, S. M. Molecular Simulation of the Concentration-Dependent In- 
teraction of Hydrophobic Drugs with Model Cellular Membranes. Journal of Physical Chemistry B 2014, 118, 11965-11972.

5. Dadwal, A.; Baldi, A.; Narang, R. K. Nanoparticles as carriers for drug delivery in cancer. Artificial Cells, Nanomedicine and Biotechnology 2018, 1 - 11.

6. Werner, M.; Auth, T.; Beales, A.; Fleury, J. B.; Hook, F.; Kress, H.; Lehn, R. C. V.; Muller, M.; Petrov, E. P.; Sarkisov, L. et al. Nanomaterial interactions with biomembranes: Bridging the gap between soft matter models and biological context. Journal of Computational Chemistry 2018, 13, 028501.

7. Walrant, A.; Cardon, S.; Burlina, F.; Sagan, S. Membrane Crossing and Membranotropic Activity of Cell-Penetrating Peptides: Dangerous Liasons? Accounts of Chemical Research 2017, 50, $2968-2975$.

8. Bennion, B. J.; Be, N. A.; McNerney, M. W.; Lao, V.; Carlson, E. M.; Valdez, C. A.; Malfatti, M. A.; Enright, H. A.; Nguyen, T. H.; Lightstone, F. C. et al. Predicting a Drug's Membrane Permeability: A Computational Model Validated With in Vitro Permeability Assay Data. Journal of Physical Chemistry B 2017, 121, 5228 - 5237.

9. Aller, S. G.; Yu, J.; Ward, A.; Weng, Y.; Chittaboina, S.; Zhuo, R.; Harell, P. M.; Trinh, Y. T.; Zhang, Q.; Urbatsch, I. L. et al. Structure of P-Glycorprotein Reveals a Molecular Basis for Poly-Specific Drug Binding. Science 2009, 323, 1718 - 1722.

10. Cho, K.; Wang, X.; Chen, Z.; Shin, D. M. Therapeutic Nanoparticles for Drug Delivery in Cancer. Clinical Cancer Research 2008, 121, 1310 - 1316.

11. Bozdaganyan, M. E.; Orekhov, P. S.; Shaytan, A. K.; Shaitan, K. V. Comparative Computational Study of Interaction of C60 Fullerene and Tris-Malonyl-C60-Fullerene Isomers with Lipid Bilayer: Relation to Their Anioxidant Effect. PLos One 2014, 9, e102487. 
12. Baoukina, S.; Mendez-Villuendas, E.; Tieleman, D. P. Molecular View of Phase Coexistence in Lipid Monolayers. Journal of the American Chemical Society 2012, 134, 17543 $-17553$.

13. London, E. New Insights into How Cholesterol and Unsaturation Control Lipid Domain Formation. Biophysical Journal 2016, 111, 465 - 466.

14. Schmid, F. Physical mechanisms of micro- and nanodomain formation in multicomponent lipid membranes. Biochimica et Biophysica Acta 2017, 1859, 509 - 528.

15. Crane, J. M.; Tamm, L. K. Role of Cholesterol in the Formation and Nature of Lipid Rafts in Planar and Spherical Model Membranes. Biophysical Journal 2004, 86, 2965 2979 .

16. Crane, J. M.; Tamm, L. K. Cholesterol Induces Uneven Curvature of Asymmetric Lipid Bilayers. Scientific World Journal 2004, 86, 2965 - 2979.

17. Ong, Q.; Luo, Z.; Stellachi, F. Characterization of Ligand Shell for Mixed-Ligand Coated Gold Nanoparticles. Accounts of Chemical Research 2017, 50, 1911 - 1919.

18. Gibson, J. D.; Khanal, B. P.; Zubarev, E. R. Paclitaxel-Functionalized Gold Nanoparticles. Journal of the American Chemical Society 2007, 129, 11653 - 11661.

19. Pengo, P.; Sologan, M.; Pasquato, L.; Guida, F.; Pacor, S.; Tossi, A.; Stellachi, F.; Marson, D.; Boccardo, S.; Pricl, S. et al. Gold nanoparticles with patterned surface monolayers for nanomedicine: current perspectives. European Biophysics Journal 2017, $46,749-771$.

20. Colangelo, E.; Comenge, J.; Paramelle, D.; Volk, M.; Chen, Q.; Levy, R. Characterizing Self-Assembled Monolayers on Gold Nanoparticles. Bioconjugate Chemistry 2017, 27, $11-22$. 
21. Angeioletti-Uberti, S. Theory, simulations and the design of functionalized nanoparticles for biomedicals applications: A Soft Matter Perspective. NPJ Computational Materials 2017, 3, $19-25$.

22. Zhang, S.; Gao, H.; Bao, G. Physical Principles of Nanoparticle Cellular Endocytosis. ACS Nano 2015, 9, 8655 - 8671.

23. Yuan, H.; Li, J.; Bao, G.; Zhang, S. Variable Nanoparticle-Cell Adhesion Strength Regulates Cellular Uptake. Physical Review Letters 2010, 105, 138101.

24. Lehn, R. C. V.; Alexander-Katz, A. Fusion of ligand-coated nanoparticles with lipid bilayers: Effect of ligand flexibility. Journal of Physical Chemistry A 2014, 118, 5848 5856.

25. Lehn, R. C. V.; Ricci, M.; Silva, P. H. J.; Andrewozzi, P.; Reguera, J.; Voitchovsky, K.; Stellachi, F.; Alexander-Katz, A. Lipid tail protrusions mediate the insertion of nanoparticles into model cell membranes. Nature Communications 2014, 5, 4482.

26. Lehn, R. C. V.; Alexander-Katz, A. Free energy change for insertion of charged, monolayer-protected nanoparticles into lipid bilayers. Soft Matter 2014, 10, 648 - 658 .

27. Jackson, A. M.; Myerson, J. W.; Stellachi, F. Spontaneous assembly of subnanometreordered domains in the ligand shell of monolayer-protected nanoparticles. Nature Materials 2004, 3, $330-336$.

28. Quan, X.; Peng, C.; Zhao, D.; Fan, J.; Zhou, J. Molecular Understanding of the Penetration of Functionlized Gold Nanoparticles into Asymmetric Membranes. Langmuir 2017, 33, $361-371$.

29. Barnoud, J.; Rossi, G.; Marrink, S. J.; Monticelli, L. Hydrophobic Compounds Reshape Membrane Domains. PLoS Computational Biology 2014, 10, e1003873. 
30. Davis, R. S.; Kumar, P. B. S.; Sperotto, M. M.; Mohamed, L. Predictions of Phase Separation in Three-Component Lipid Membranes by the MARTINI Force Field. Journal of Physical Chemistry B 2013, 117, 4072 - 4080.

31. Hakobyan, D.; Heuer, A. Key Molecular Requirements for Raft Formation for Lipid/Cholestrol Membranes. PLoS ONE 2014, 9, e87369.

32. Jewell, C. M.; Jung, J.-M.; Atukorale, P. U.; Carney, P. R.; Stellachi, F.; Irvine, D. J. Angewandte Chemie International Edition. Oligonucleotide Delivery by Cell-Penetrating "Striped" Nanoparticles 2011, 50, 12312-12315.

33. Berendsen, H. J. C.; van der Spoel, D.; van Drunen, R. GROMACS: A message-passing parallel molecular dynamics implementation. Computer Physics Communications 1995, $91,43-56$.

34. Abraham, M. J.; Murtola, T.; Schulz, R.; Pall, S.; Smith, J. C.; Hess, B.; Lindahl, E. GROMACS: High performance molecular simulations through multi-level parallelism from laptops to supercomputers. SoftwareX 2015, 1-2, $19-25$.

35. Parrinello, M.; Rahman, A. Crystal Structure and Pair Potentials: A MolecularDynamics Study. Physical Review Letters 1980, 45, 1196 - 1199.

36. Parrinello, M.; Rahman, A. Polymorphic transitions in single crystals: A new molecular dynamics method. Journal of Applied Physics 1981, 52, 7182 - 1199.

37. Parrinello, M.; Rahman, A. Strain fluctuations and elastic constants. Journal of Chemical Physics 1982, 76, 2662.

38. Risselada, H. J.; Marrink, S. J. The molecular face of lipid rafts in model membranes. Proceedings of the National Academy of Sciences of the United States of America 2008, 105, $17367-17372$. 
39. Chen, X.; Tieleman, D. P.; Liang, Q. Modulating interactions between ligand-coated nanoparticles and phase separated lipid bilayers by varying the ligand density and the surface charge. Nanoscale 2018, 10, 2481 - 2491.

40. The MARTINI Force Field: Coarse Grained Model for Biomolecular Simulations. Journal of Physical Chemistry B 2007, 111, 7812 - 7824.

41. Wassenar, T. A.; Ingolfsson, H. I.; Bockmann, R. A.; Tieleman, D. P.; Marrink, S. J. Computational Lipidomics with insane: A Versatile Tool for Generating Custom Membranes for Molecular Simulations. Journal of Chemical Theory Computation 2015, 11, $2144-2155$.

42. Martinez, L.; Andrade, A.; Birgin, E. H.; Martinez, J. M. Journal of Computational Chemistry. Packmol: A package for building initial configurations for molecular dynamics simulations 2009, 30, 2157-2164.

43. MartẪynez, J. M.; MartÃynez, L. Journal of Computational Chemistry. Packing optimization for automated generation of complex system's initial configurations for molecular dynamics and docking 2003, 24, 819-825.

44. Ge, X.; Ke, P. C.; Davis, T. P.; Ding, F. A Thermodynamics Model for the Emergence of a Stripe-like Bnary SAM on a Nanoparticle Surface. Small 2015, 11, 4894 - 4899.

45. Simonelli, F.; Bochicchio, D.; Ferrando, R.; Rossi, G. Monolayer-Protected Anionic Au Nanoparticles Walk into Lipid Membranes Step by Step. Journal of Physical Chemistry Letters 2015, 6, $3175-3179$.

46. Gkeka, P.; Angelikopoulos, P.; Sarkisov, L.; Cournia, Z. Membrane Partitioning of Anionic, Ligand-Coated Nanoparticles is Accompanied by Ligand Snorkelling, Local Disordering, and Cholestrol Depletion. PloS Computational Biology 2014, 10, e1003917. 
47. Angeliopoulos, P.; Sarkisov, L.; Cournia, Z.; Gkeka, P. Self-assembly of anionic, ligandcoated nanoparticles in lipid membranes. Nanoscale 2017, 9, 1040 - 1048.

48. Frenkel, D.; Smit, B. Understanding Molecular Simulation; 2002.

49. Rapaport, D. C. The Art of Molecular Simulation; 2004.

50. Verlet, L. Computer Experiments on Classical Fluids. I. Thermodynamical Properties of Lennard-Jones Molecules. Physical Review 1962, 159, 98 - 103.

51. Swope, C. W.; Andersen, H. C.; Berens, P. H.; Wilson, K. R. A computer simulation method for the calculation of equilibrium constants for the formation of physical clusters of molecules: Application to small water clusters. Journal of Chemical Physics 1982, $76,637$.

52. Castillo, N.; Monticelli, L.; Barnoud, J.; Tieleman, D. P. Free Energy of WALP23 Dimer Association in DMPC, DPPC, and DOPC bilayers. Chemistry and Physics of Lipids 2013, 169, $95-105$.

53. Vanegas, J. M.; Torres-Sanchez, A.; Arroyo, M. Importance of Force Decomposition for Local Stress Calculations in Biomembrane Molecular Simulations. Journal of Chemical Theory Computation 2014, 10, 691 - 702.

54. Torres-Sanchez, A.; Vanegas, J. M.; Arroyo, M. Examining the Mechanical Equilibrium of Microscopic Stresses in Molecular Simulations. Physical Review Letters 2015, 114, 258102.

55. Katira, S.; Mandadapu, K. K.; Vaikuntanathan, S.; Smit, B.; Chandler, D. Pre-transition effects mediate forces of assembly between transmembrane proteins. eLife 2015, 11, 13150.

56. Nelson, D. R.; Halperin, B. I. Theory of two-dimensional melting. Physical Review Letters 1978, 41, $121-124$. 
57. Nelson, D. R.; Halperin, B. I. Dislocation-mediated melting in two dimensions. Physical Review B 1979, 19, $2457-2484$.

58. Pantelopoulos, G. A.; Nagai, T.; Bandara, A.; Panahi, A.; Straub, J. E. Critical size dependence of domain formation observed in coarse-grained simulations of bilayers composed of ternary lipid mixtures. Journal of Chemical Physics 2017, 7, 095101.

59. Humphrey, W.; Dalke, A.; Schulten, K. VMD - Visual Molecular Dynamics. Journal of Molecular Graphics 1996, 14, 33 - 38.

60. Lukat, G.; Kruger, J.; Sommer, B. APL@Voro: A Voronoi-Based Membrane Analysis Tool for GROMACS Trajectories. Journal of Chemical Information and Modeling 2013, $53,2908-2925$.

61. Michaud-Agrawal, N.; Denning, E. J.; Woolf, T. B.; ; Beckstein, O. MDAnalysis: A Toolkit for the Analysis of Molecular Dynamics Simulations. Journal of Computational Chemistry 2011, 32, 2319 - 2327.

62. Hu, S.; Huang, C.; Tsao, H.; Sheng, Y. Hybrid membranes of lipids and diblock copolymers: From homogenity to rafts to phase separation. Physical Review E 2019, 99, 012403.

63. Schafer, L. V.; de Jong, D. H.; Holt, A.; Rzepiela, A. J.; de Vries, A. H.; Poolman, B.; Killian, J. A.; Marrink, S. J. Lipid packing drives the segregation of transmembrane helices into disordered lipid domains in model membranes. Proceedings of the National Academy of Sciences of the United States of America 2011, 108, 1343 - 1348.

64. Parton, D. L.; Klingelhoefer, J. W.; Sansom, M. S. P. Aggregation of Model Membrane Proteins, Modulated by Hydrophobic Mismatch, Membrane Curvature, and Protein Class. Biophysical Journal 2011, 101, 691 - 699. 
65. Madsen, J. J.; Grime, J. M. A.; Rossman, J. S.; Voth, G. A. Entropic forces drive clustering and spatial localization of influenza A M2 during viral budding. Proceedings of the National Academy of Sciences of the United States of America 2018, 115, E8595 - E8603.

66. Parton, D. L.; Tek, A.; Baaden, M.; Sansom, M. S. P. Formation of Raft-like Assemblies within Custers of Influenza Hemagglutin Observed by MD Simulations. PLoS Compututational Biology 2013, 9, e1003034.

67. Ollila, O. H. S.; Risselada, H. J.; Louhivouri, M.; Lindahl, R. 3D Pressure Field in Lipid Membranes and Membrane-Protein Complexes. Physical Review Letters 2009, 102, 078101.

68. Yefimov, S.; van der Giesson, R.; Onck, P. R.; Marrink, S. J. Mechanosensitive Membrane Channels in Action. Biophysical Journal 2008, 94, 2994 - 3002.

69. Yoo, J.; Cui, Q. Membrane-Mediated Protein-Protein Interactions and Connection to Elastic Models: A Coarse-Grained Simulation Analysis of Gramicidin A Association. Biophysical Journal 2013, 104, 128 - 138.

70. Katira, S. Physical Considerations of the Organization of Inclusions in Lipid Bilayer Systems. Ph.D. thesis, University of California, Berkeley, 2015. 


\section{Graphical TOC Entry}

Some journals require a graphical entry for the Table of Contents. This should be laid out "print ready" so that the sizing of the text is correct. Inside the tocentry environment, the font used is Helvetica $8 \mathrm{pt}$, as required by Journal of the American Chemical Society.

The surrounding frame is $9 \mathrm{~cm}$ by $3.5 \mathrm{~cm}$, which is the maximum permitted for Journal of the American Chemical Society graphical table of content entries. The box will not resize if the content is too big: instead it will overflow the edge of the box.

This box and the associated title will always be printed on a separate page at the end of the document. 